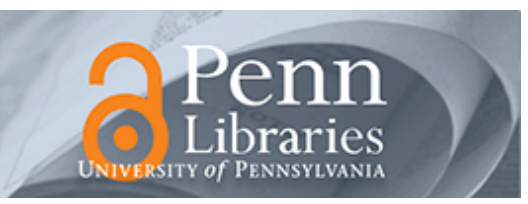

University of Pennsylvania

ScholarlyCommons

June 2007

\title{
Statistical characteristics of the envelope in diversity combining of two correlated Rayleigh fading channels
}

\author{
W Tang \\ University of Pennsylvania \\ Saleem Kassam \\ University of Pennsylvania, kassam@seas.upenn.edu
}

Follow this and additional works at: https://repository.upenn.edu/ese_papers

\author{
Recommended Citation \\ W Tang and Saleem Kassam, "Statistical characteristics of the envelope in diversity combining of two \\ correlated Rayleigh fading channels", . June 2007.
}

Copyright 2007 IEEE. Reprinted from IET Communications, Volume 1, Issue 3, June 2007, pages 405-413.

This material is posted here with permission of the IEEE. Such permission of the IEEE does not in any way imply IEEE endorsement of any of the University of Pennsylvania's products or services. Internal or personal use of this material is permitted. However, permission to reprint/republish this material for advertising or promotional purposes or for creating new collective works for resale or redistribution must be obtained from the IEEE by writing to pubs-permissions@ieee.org. By choosing to view this document, you agree to all provisions of the copyright laws protecting it.

This paper is posted at ScholarlyCommons. https://repository.upenn.edu/ese_papers/307

For more information, please contact repository@pobox.upenn.edu. 


\title{
Statistical characteristics of the envelope in diversity combining of two correlated Rayleigh fading channels
}

\begin{abstract}
Performance of diversity systems is often evaluated under the assumption of perfect interleaving and characterised in terms of long-term parameters such as the average bit-error rate, which does not capture the dynamics of fading channels. Statistical characteristics (static and dynamic) of the envelope of two correlated Rayleigh fading channels are explored using a physical model. For two popular diversitycombining schemes, maximal ratio combining and selection combining, both static and dynamic (levelcrossing rate) properties of correlated fading channels are derived. These results are very useful for performance evaluation of diversity systems without bit-level simulations. The results can also provide very useful characteristics such as average duration of fades, fading rate and outage probability for twochannel diversity systems and can be extended to multiple fading channels.

\section{Comments}

Copyright 2007 IEEE. Reprinted from IET Communications, Volume 1, Issue 3, June 2007, pages 405-413.

This material is posted here with permission of the IEEE. Such permission of the IEEE does not in any way imply IEEE endorsement of any of the University of Pennsylvania's products or services. Internal or personal use of this material is permitted. However, permission to reprint/republish this material for advertising or promotional purposes or for creating new collective works for resale or redistribution must be obtained from the IEEE by writing to pubs-permissions@ieee.org. By choosing to view this document, you agree to all provisions of the copyright laws protecting it.
\end{abstract}




\title{
Statistical characteristics of the envelope in diversity combining of two correlated Rayleigh fading channels
}

\author{
W. Tang and S.A. Kassam
}

\begin{abstract}
Performance of diversity systems is often evaluated under the assumption of perfect interleaving and characterised in terms of long-term parameters such as the average bit-error rate, which does not capture the dynamics of fading channels. Statistical characteristics (static and dynamic) of the envelope of two correlated Rayleigh fading channels are explored using a physical model. For two popular diversity-combining schemes, maximal ratio combining and selection combining, both static and dynamic (level-crossing rate) properties of correlated fading channels are derived. These results are very useful for performance evaluation of diversity systems without bit-level simulations. The results can also provide very useful characteristics such as average duration of fades, fading rate and outage probability for two-channel diversity systems and can be extended to multiple fading channels.
\end{abstract}

\section{Introduction}

Effect and mitigation of fading is of prime concern in wireless transmission $[1,2]$. The performance of wireless links is often evaluated under an assumption of perfect interleaving and characterised in terms of long-term parameters such as the average bit-error rate (BER). To capture the dynamic characteristics of a fading channel, the joint probability density function (pdf) $p\left(r_{1}, \dot{r}_{1}\right)$ of the fading envelope $r_{1}$ and its derivative $\dot{r}_{1}$ may be used [2]. From this, information may be obtained on parameters such as level-crossing rate (LCR), average fade duration and outage probability, and used to evaluate more explicitly the performance of wireless links. In $[3,4]$, this approach was used to characterise performance of a single-path transmission link.

Various diversity schemes have been used as countermeasures for fading in wireless links. These include spatial (path) diversity and frequency diversity. Two commonly used diversity combining schemes are maximal ratio combining (MRC) and selection combining. For the evaluation of diversity-systems performance, it is typically assumed that the fading envelopes of diversity branches are independent of each other, but in practice they may be correlated. Examples of performance analyses of diversity system for correlated fading channels can be found in [5]. However, these analyses under such fading are based on long-term averages such as the average BER. For some systems [e.g. systems that utilise an automatic-repeatrequest (ARQ) scheme], the system performance depends on the dynamics of the fading channel. The long-term

(C) The Institution of Engineering and Technology 2007

doi:10.1049/iet-com:20050506

Paper first received 24th November 2004 and in revised form 16th July 2006 W. Tang is with the Department of Electrical and Systems Engineering, University of Pennsylvania, Philadelphia, PA 19104, USA and also with Snowbell Court 2, E Brunswick, NJ 08816, USA

S.A. Kassam is with the Department of Electrical and Systems Engineering, University of Pennsylvania, Philadelphia, PA 19104, USA

E-mail: wtang0327@yahoo.com average BER performance is then not adequate for performance analysis. Procedures for generating correlated fading envelopes are discussed in [6] and can be used to perform bit-level performance simulations. However, bit-level simulations of the performance can be very time-consuming and do not lead to a fundamental understanding of dependence of performance on the fading dynamics.

In this paper, we explore both the static and dynamic statistical characteristics of the envelope resulting from diversity combining of two correlated Rayleigh fading signals. Specifically, we obtain the joint pdf $p\left(r_{1}, r_{2}, \dot{r}_{1}, \dot{r}_{2}\right)$ for the fading envelopes and their derivatives, and then derive LCR and average fade duration of the diversity-combined signal envelope. The results we obtain are useful in characterising the statistical properties of a signal obtained from a diversity-combining scheme such as MRC or selection combing. These statistical characterisations can be used to obtain more detailed performance characteristics for diversity reception over fading channels in specific cases. For example, an ARQ scheme may be analysed for performance using the dynamics of the diversity combined signal. This is facilitated through the use of a finite-state Markov model, as in $[3,4]$ for the single-fading channel with no diversity. The results in this paper can be used to construct finite-state Markov models for correlated-path diversity schemes and applied to analyse the performance of ARQ schemes [7].

To derive the joint pdf $p\left(r_{1}, r_{2}, \dot{r}_{1}, \dot{r}_{2}\right)$, we construct a physical model to investigate the covariance between the components of two correlated Rayleigh fading envelopes. We can then obtain the covariance matrix of the four Gaussian components and their derivatives for the two correlated Rayleigh fading envelopes. From this, we are able to derive the pdf $p\left(r_{1}, r_{2}, \dot{r}_{1}, \dot{r}_{2}\right)$ of the two correlated fading envelopes and their derivatives. We then consider specifically two popular diversity combining schemes. First the MRC, in which the receiver combines the diversity branches by weighting them using the complex conjugates of their respective signal amplitudes before adding them. Therefore the 'total received power' from these diversity branches determines the performance of the diversity 
system. Second, the 'selection combining', in which the receiver chooses the branch with maximum received power for use in signal detection. Hence, the 'maximum power' from these diversity branches determines the performance of this diversity system.

We show how $p\left(r_{1}, r_{2}, \dot{r}_{1}, \dot{r}_{2}\right)$ can be used to obtain useful statistics such as LCR and average fade duration for the total power and maximum power. This is done through the joint pdf for the random process of total or maximum power and its derivative. Then, we can find LCR for total power and maximum power using level-crossing analysis for a single random process, and we can also obtain the average fade duration of total power and maximum power. Related work is presented in [8], in which a different approach is used to obtain the LCR in MRC. The development in [8] starts with independent envelopes and extends the results using an eigendecomposition of the envelope correlation matrix. Our LCR results are functions of the cross-correlation coefficient of fading envelopes. Our approach starts from a physical model and uses the correlation function of the fading envelopes directly, and we obtain explicit results, that can be directly interpreted and applied in performance analysis. The results in [8] are for the LCR for the case of MRC only and are expressed as functions of the eigenvalues of a correlation matrix for which there is no explicit physical model. Without a physical model, it is difficult to apply these results because the correlation matrix and its eigenvalues must be available.

We have shown the validity of our statistical results for two correlated fading channels in [7], through simulations generated using the method described in [6]. The preliminary work of Tang and Kassam [7] also gives a finite-state Markov model representing correlated fading channels and demonstrates its use in analysing the performance of ARQ schemes.

\section{Physical model for two correlated fading channels}

\subsection{Received signal}

A multipath model for two correlated Rayleigh fading channels in spatial diversity is shown in Fig. 1. The same transmitted signal $A \exp \left[j\left(\omega_{1} t+\psi_{1}\right)\right]$ is reflected by $N$ scatterers, and then received by two receivers $R_{1}$ and $R_{2}$. The two receivers are separated by a distance $d$. $A$ is the amplitude of the transmitted signal, $\omega_{1}$ the carrier frequency and $\psi_{1}$ the unknown phase of the transmitted signal. We assume that $\psi_{1}$ is uniformly distributed on $[0,2 \pi]$. The transmitted signal arrives at the $n$th scatterer with a phase $\phi_{n}$, which depends on the distance between the transmitter and the $n$th scatterer. We assume that the $\phi_{n}$ are independent of each other and of $\psi_{1}$, and $\phi_{n}$ are uniformly distributed on $[0,2 \pi] . n=1,2, \ldots, N$.

Let $\alpha_{n}, T_{n}$ and $\theta_{n}$ be the set of parameters associated with the $n$th path for $R_{1}$, where $\alpha_{n}$ is the attenuation factor. $T_{n}$ is the time delay for the path, and $\theta_{n}$ is the angle between the receiver's direction of motion and the direction of arrival of the $n$th path signal (Figs. 1 and 2). The $n$th delayed signal component, arriving at an angle $\theta_{n}$, will contribute to a Doppler shift equal to

$$
\omega_{d_{n}}=\omega_{d} \cos \theta_{n}
$$

where $\omega_{d}=2 \pi f_{\mathrm{m}}$ and $f_{\mathrm{m}}$ is the maximum Doppler frequency, $v / \lambda$. Here $\lambda$ is the wavelength corresponding to

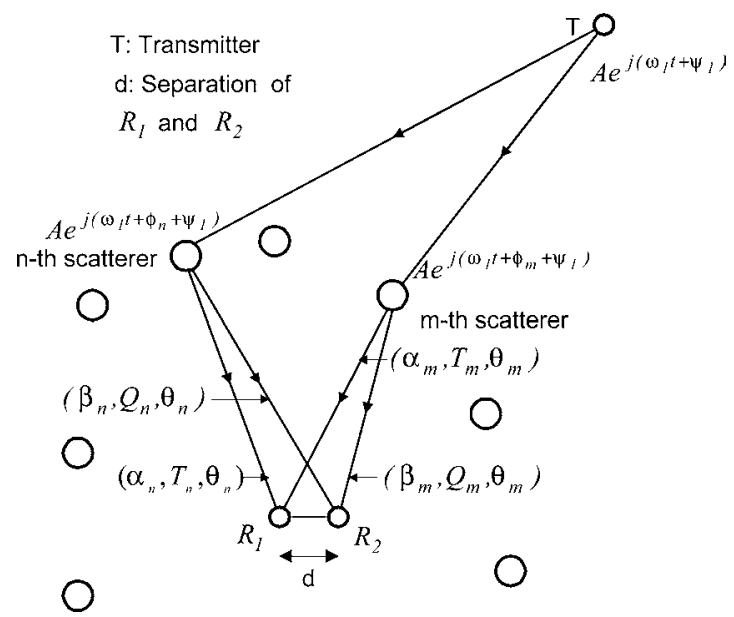

Fig. 1 Multipath channel model for spatial diversity

the transmitted carrier frequency, and $v$ is the speed of the receiver. The received complex waveform at the first receiver is

$$
\begin{aligned}
& A \sum_{n=1}^{N} \alpha_{n} \mathrm{e}^{j\left[\left(\omega_{1}+\omega_{d_{n}}\right)\left(t-T_{n}\right)+\phi_{n}+\psi_{1}\right]} \\
& \quad=A \sum_{n=1}^{N} \alpha_{n} \mathrm{e}^{j\left(\omega_{1} t+\omega_{d_{n}}\left(t-T_{n}\right)-\omega_{1} T_{n}+\phi_{n}+\psi_{1}\right)}
\end{aligned}
$$

The real part of the received signal is [2]

$$
R_{1}(t)=X_{1} \cos \omega_{1} t-Y_{1} \sin \omega_{1} t
$$

where

$$
\begin{aligned}
& X_{1}=A \sum_{n=1}^{N} \alpha_{n} \cos \left(\omega_{d_{n}}\left(t-T_{n}\right)-\omega_{1} T_{n}+\phi_{n}+\psi_{1}\right) \\
& Y_{1}=A \sum_{n=1}^{N} \alpha_{n} \sin \left(\omega_{d_{n}}\left(t-T_{n}\right)-\omega_{1} T_{n}+\phi_{n}+\psi_{1}\right)
\end{aligned}
$$

The two receivers are separated by a distance $d$. We assume that the separation $d$ of the receivers is very small compared with the distance between the scatterer and the receiver. Thus, the angle of incidence $\theta_{n}$ for the $n$th path, as shown in Fig. 2, is the same for the two receivers. Let

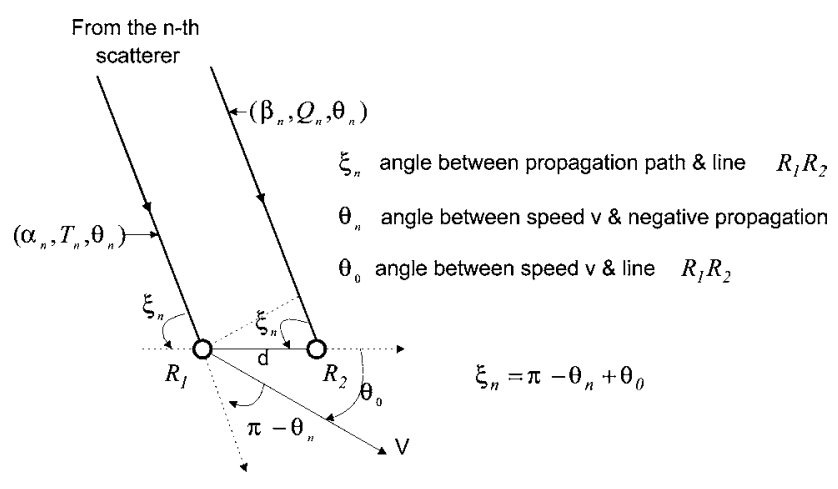

Fig. 2 Multipath channel model for spatial diversity

Illustration of the angle $\theta_{0}$ between direction of motion and orientation of receivers 
$\left(\beta_{n}, Q_{n}, \theta_{n}\right)$ be the amplitude, time delay and the angle associated with the $n$th path for receiver $R_{2}$, respectively. Then, the in-phase and quadrature components at $R_{2}$ are

$$
\begin{aligned}
& X_{2}=A \sum_{n=1}^{N} \beta_{n} \cos \left(\omega_{d_{n}}\left(t-Q_{n}\right)-\omega_{1} Q_{n}+\phi_{n}+\psi_{1}\right) \\
& Y_{2}=A \sum_{n=1}^{N} \beta_{n} \sin \left(\omega_{d_{n}}\left(t-Q_{n}\right)-\omega_{1} Q_{n}+\phi_{n}+\psi_{1}\right)
\end{aligned}
$$

From $((4 a))-((4 d))$, we find the corresponding derivatives of the four components to be

$$
\begin{aligned}
\dot{X}_{1}= & \omega_{d} A \sum_{n=1}^{N}-\alpha_{n} \sin \left(\omega_{d_{n}}\left(t-T_{n}\right)\right. \\
& \left.-\omega_{1} T_{n}+\phi_{n}+\psi_{1}\right) \cos \theta_{n} \\
\dot{Y}_{1}= & \omega_{d} A \sum_{n=1}^{N} \alpha_{n} \cos \left(\omega_{d_{n}}\left(t-T_{n}\right)\right. \\
& \left.-\omega_{1} T_{n}+\phi_{n}+\psi_{1}\right) \cos \theta_{n} \\
\dot{X}_{2}= & \omega_{d} A \sum_{n=1}^{N}-\beta_{n} \sin \left(\omega_{d_{n}}\left(t-Q_{n}\right)\right. \\
& \left.-\omega_{1} Q_{n}+\phi_{n}+\psi_{1}\right) \cos \theta_{n} \\
\dot{Y}_{2}= & \omega_{d} A \sum_{n=1}^{N} \beta_{n} \cos \left(\omega_{d_{n}}\left(t-Q_{n}\right)\right. \\
& \left.-\omega_{1} Q_{n}+\phi_{n}+\psi_{1}\right) \cos \theta_{n}
\end{aligned}
$$

Note that the differential delay $\tau_{n}=Q_{n}-T_{n}$ depends on the orientation of the line connecting $R_{1}$ and $R_{2}$. We will use this later in obtaining the covariance matrix.

\subsection{Covariance between components of two equal-power fading envelopes}

We define the eight-component vector $\boldsymbol{Z}=\left(X_{1}, Y_{1}, \dot{X}_{1}, \dot{Y}_{1}\right.$, $\left.X_{2}, Y_{2}, \dot{X}_{2}, \dot{Y}_{2}\right)^{\mathrm{T}}$, which contains the $I$, Q components and component derivatives at the two receivers. We now discuss the mean value and covariance matrix for $\boldsymbol{Z}$. The mean value of each of these eight random variables is zero. This is because the random phases $\phi_{n}$ are uniformly distributed in $[0,2 \pi]$. Since these eight random variables have zero mean, we have $\operatorname{Cov}\left[G_{j}, G_{k}\right]=E\left[G_{j} G_{k}\right]$, where $G_{j}$ and $G_{k}$ represent any two of these eight random variables.

Now we assume (i) the number $N$ of scatterers is large, and (ii) the scattering amplitudes $\alpha_{n}$ and $\beta_{n}$ are sequences of independent random amplitudes with non-vanishing and bounded moments of order up to three. Then we can approximate the $X_{1}, Y_{1}, X_{2}$ and $Y_{2}$ as jointly Gaussian random variables with zero means. We can also easily show that $X_{1}$ and $Y_{1}$ are uncorrelated, and therefore independent under the Gaussian approximation, with equal variances. Therefore the envelope $r_{1}=\left(X_{1}^{2}+Y_{1}^{2}\right)$ is assumed to have a Rayleigh distribution. Similarly, the second envelope $r_{2}=\left(X_{2}^{2}+Y_{2}^{2}\right)$ has a Rayleigh distribution. However, $r_{1}$ and $r_{2}$ are in general correlated.

Using (4a)-(4h), we can obtain the pairwise covariances for the eight variables in $\boldsymbol{Z}$. The zero covariance matrix elements turn out to be [we obtain $(5 b)$ and $(5 c)$ with an assumption of uniform scatterer]

$$
\begin{aligned}
\operatorname{Cov}\left[X_{1}, Y_{1}\right] & =\operatorname{Cov}\left[X_{2}, Y_{2}\right]=0 \\
\operatorname{Cov}\left[X_{1}, \dot{Y}_{1}\right] & =\operatorname{Cov}\left[\dot{X}_{1}, Y_{1}\right]=0 \\
\operatorname{Cov}\left[X_{2}, \dot{Y}_{2}\right] & =\operatorname{Cov}\left[\dot{X}_{2}, Y_{2}\right]=0 \\
\operatorname{Cov}\left[\dot{X}_{1}, \dot{Y}_{1}\right] & =\operatorname{Cov}\left[\dot{X}_{2}, \dot{Y}_{2}\right]=0 \\
\operatorname{Cov}\left[X_{1}, \dot{X}_{1}\right] & =\operatorname{Cov}\left[Y_{1}, \dot{Y}_{1}\right]=\operatorname{Cov}\left[X_{2}, \dot{X}_{2}\right] \\
& =\operatorname{Cov}\left[Y_{2}, \dot{Y}_{2}\right]=0 \\
\operatorname{Cov}\left[\dot{X}_{1}, X_{1}\right] & =\operatorname{Cov}\left[\dot{Y}_{1}, Y_{1}\right]=\operatorname{Cov}\left[\dot{X}_{2}, X_{2}\right] \\
& =\operatorname{Cov}\left[\dot{Y}_{2}, Y_{2}\right]=0
\end{aligned}
$$

The other covariance matrix elements come from 13 possible non-zero values (including four pairs of \pm values), which are as follows

$$
\begin{aligned}
& \operatorname{Cov}\left[X_{1}, X_{1}\right]=\operatorname{Cov}\left[X_{2}, X_{2}\right]=\operatorname{Cov}\left[Y_{1}, Y_{1}\right] \\
& =\operatorname{Cov}\left[Y_{2}, Y_{2}\right] \triangleq \sigma^{2} \\
& \operatorname{Cov}\left[\dot{X}_{1}, \dot{X}_{1}\right]=\operatorname{Cov}\left[\dot{Y}_{1}, \dot{Y}_{1}\right] \triangleq \dot{\sigma}_{1}^{2} \\
& \operatorname{Cov}\left[\dot{X}_{2}, \dot{X}_{2}\right]=\operatorname{Cov}\left[\dot{Y}_{2}, \dot{Y}_{2}\right] \triangleq \dot{\sigma}_{2}^{2} \\
& \operatorname{Cov}\left[X_{1}, X_{2}\right]=\operatorname{Cov}\left[X_{2}, X_{1}\right]=\operatorname{Cov}\left[Y_{1}, Y_{2}\right] \\
& =\operatorname{Cov}\left[Y_{2}, Y_{1}\right] \triangleq \mu_{1} \\
& \operatorname{Cov}\left[X_{1}, Y_{2}\right]=\operatorname{Cov}\left[Y_{2}, X_{1}\right]=-\operatorname{Cov}\left[Y_{1}, X_{2}\right] \\
& =-\operatorname{Cov}\left[X_{2}, Y_{1}\right] \triangleq \mu_{2} \\
& \operatorname{Cov}\left[X_{1}, \dot{X}_{2}\right]=\operatorname{Cov}\left[\dot{X}_{2}, X_{1}\right]=\operatorname{Cov}\left[Y_{1}, \dot{Y}_{2}\right] \\
& =\operatorname{Cov}\left[\dot{Y}_{2}, Y_{1}\right]=-\operatorname{Cov}\left[\dot{X}_{1}, X_{2}\right] \\
& =-\operatorname{Cov}\left[X_{2}, \dot{X}_{1}\right]=-\operatorname{Cov}\left[\dot{Y}_{1}, Y_{2}\right] \\
& =-\operatorname{Cov}\left[Y_{2}, \dot{Y}_{1}\right] \triangleq \mu_{3} \\
& \operatorname{Cov}\left[X_{1}, \dot{Y}_{2}\right]=\operatorname{Cov}\left[\dot{Y}_{2}, X_{1}\right]=-\operatorname{Cov}\left[Y_{1}, \dot{X}_{2}\right] \\
& =-\operatorname{Cov}\left[\dot{X}_{2}, Y_{1}\right]=-\operatorname{Cov}\left[\dot{X}_{1}, Y_{2}\right] \\
& =-\operatorname{Cov}\left[Y_{2}, \dot{X}_{1}\right]=\operatorname{Cov}\left[\dot{Y}_{1}, X_{2}\right] \\
& =\operatorname{Cov}\left[X_{2}, \dot{Y}_{1}\right] \triangleq \mu_{4} \\
& \operatorname{Cov}\left[\dot{X}_{1}, \dot{X}_{2}\right]=\operatorname{Cov}\left[\dot{X}_{2}, \dot{X}_{1}\right]=\operatorname{Cov}\left[\dot{Y}_{1}, \dot{Y}_{2}\right] \\
& =\operatorname{Cov}\left[\dot{Y}_{2}, \dot{Y}_{1}\right] \triangleq \mu_{5} \\
& \operatorname{Cov}\left[\dot{X}_{1}, \dot{Y}_{2}\right]=\operatorname{Cov}\left[\dot{Y}_{2}, \dot{X}_{1}\right]=-\operatorname{Cov}\left[\dot{Y}_{1}, \dot{X}_{2}\right] \\
& =-\operatorname{Cov}\left[\dot{X}_{2}, \dot{Y}_{1}\right] \triangleq \mu_{6}
\end{aligned}
$$

The set of parameters $\sigma^{2}, \dot{\sigma}_{1}^{2}, \dot{\sigma}_{2}^{2}, \mu_{1}, \mu_{2}, \mu_{3}, \mu_{4}, \mu_{5}$ and $\mu_{6}$ is given by

$$
\sigma^{2}=\frac{A^{2}}{2} \times E\left[\sum_{n=1}^{N} \alpha_{n}^{2}\right]=\frac{A^{2}}{2} \times E\left[\sum_{n=1}^{N} \beta_{n}^{2}\right]
$$


$\dot{\sigma}_{l}^{2}=\frac{A^{2} \omega_{d}^{2}}{2} \times E\left[\sum_{n=1}^{N} \alpha_{n}^{2} \cos ^{2} \theta_{n}\right]$

$\dot{\sigma}_{2}^{2}=\frac{A^{2} \omega_{d}^{2}}{2} \times E\left[\sum_{n=1}^{N} \beta_{n}^{2} \cos ^{2} \theta_{n}\right]$

$\mu_{1}=\frac{A^{2}}{2} \times E\left[\sum_{n=1}^{N} \alpha_{n} \beta_{n} \cos \left(\left(\omega_{d_{n}}+\omega_{1}\right) \tau_{n}\right)\right]$

$\mu_{2}=-\frac{A^{2}}{2} \times E\left[\sum_{n=1}^{N} \alpha_{n} \beta_{n} \sin \left(\left(\omega_{d_{n}}+\omega_{1}\right) \tau_{n}\right)\right]$

$\mu_{3}=\frac{A^{2} \omega_{d}}{2} \times E\left[\sum_{n=1}^{N} \alpha_{n} \beta_{n} \sin \left(\left(\omega_{d_{n}}+\omega_{1}\right) \tau_{n}\right) \cos \theta_{n}\right]$

$\mu_{4}=\frac{A^{2} \omega_{d}}{2} \times E\left[\sum_{n=1}^{N} \alpha_{n} \beta_{n} \cos \left(\left(\omega_{d_{n}}+\omega_{1}\right) \tau_{n}\right) \cos \theta_{n}\right]$

$\mu_{5}=\frac{A^{2} \omega_{d}^{2}}{2} \times E\left[\sum_{n=1}^{N} \alpha_{n} \beta_{n} \cos \left(\left(\omega_{d_{n}}+\omega_{1}\right) \tau_{n}\right) \cos ^{2} \theta_{n}\right]$

$\mu_{6}=-\frac{A^{2} \omega_{d}^{2}}{2} \times E\left[\sum_{n=1}^{N} \alpha_{n} \beta_{n} \sin \left(\left(\omega_{d_{n}}+\omega_{1}\right) \tau_{n}\right) \cos ^{2} \theta_{n}\right]$

where $\omega_{d}$ and $\omega_{d_{n}}$ are given by (1), and

$$
\tau_{n}=Q_{n}-T_{n}
$$

2.2.1 Covariance matrix $\Lambda$ : From $(5 a)-(5 o)$, the $(8 \times 8)$ covariance matrix $\boldsymbol{\Lambda}$ of the Gaussian components and component derivatives of the two Rayleigh fading channels can be written as

$$
\begin{aligned}
\boldsymbol{\Lambda} & =E\left\{\boldsymbol{Z}^{\mathrm{T}}\right\} \\
& =\left[\begin{array}{cccccccc}
\sigma^{2} & 0 & 0 & 0 & \mu_{1} & \mu_{2} & \mu_{3} & \mu_{4} \\
0 & \sigma^{2} & 0 & 0 & -\mu_{2} & \mu_{1} & -\mu_{4} & \mu_{3} \\
0 & 0 & \dot{\sigma}_{1}^{2} & 0 & -\mu_{3} & -\mu_{4} & \mu_{5} & \mu_{6} \\
0 & 0 & 0 & \dot{\sigma}_{1}^{2} & \mu_{4} & -\mu_{3} & -\mu_{6} & \mu_{5} \\
\mu_{1} & -\mu_{2} & -\mu_{3} & \mu_{4} & \sigma^{2} & 0 & 0 & 0 \\
\mu_{2} & \mu_{1} & -\mu_{4} & -\mu_{3} & 0 & \sigma^{2} & 0 & 0 \\
\mu_{3} & -\mu_{4} & \mu_{5} & -\mu_{6} & 0 & 0 & \dot{\sigma}_{2}^{2} & 0 \\
\mu_{4} & \mu_{3} & \mu_{6} & \mu_{5} & 0 & 0 & 0 & \dot{\sigma}_{2}^{2}
\end{array}\right]
\end{aligned}
$$

where $\sigma^{2}, \dot{\sigma}_{1}^{2}, \dot{\sigma}_{2}^{2}, \mu_{1}, \mu_{2}, \mu_{3}, \mu_{4}, \mu_{5}$ and $\mu_{6}$ are defined in $(5 \mathrm{~g})-(5 \mathrm{o})$. The covariance matrix has an interesting block structure with diagonal and symmetric blocks.

\subsection{Evaluation of covariance between components of two fading envelopes}

Each element in the covariance matrix $\boldsymbol{\Lambda}$ can be written as a function of the basic parameters of the model, and explicit results will allow us to obtain the joint pdf $p\left(r_{1}, r_{2}, \dot{r}_{1}, \dot{r}_{2}\right)$ for the correlated fading envelopes and their time derivatives. We are able to compute the expectations in (6a)(6i) for the covariance matrix elements under the following three assumptions: $(i)$ the distances between receivers and scatterers are much larger than the separation $d$ of the receivers, $(i i)$ the scatterers are located around the receivers, with incident power to the receivers arriving uniformly from all directions $[0,2 \pi]$ and (iii) $\alpha_{n}=\beta_{n}$. We discuss some extensions in [9].

As an example, consider the evaluation of covariance element $\mu_{5}$ of $(5 \mathrm{n})$ and ( $\left.6 \mathrm{~h}\right)$. We start evaluating $\mu_{5}$ by examining the phase term $\left(\omega_{d_{n}}+\omega_{1}\right) \tau_{n}$ in $(6 \mathrm{~h})$. Note that usually

$$
\left(\omega_{1}+\omega_{d_{n}}\right) \tau_{n} \simeq \omega_{1} \tau_{n}
$$

This is because in most cases $\omega_{d} \tau_{n}$ is very small compared to $2 \pi$. For example, for a speed $50 \mathrm{~km} / \mathrm{h}$ and carrier frequency $1.9 \mathrm{GHz}$, the maximum Doppler shift $f_{\mathrm{m}}=\omega_{d} / 2 \pi$ is $88 \mathrm{~Hz}$. Also note that $\tau_{n}=Q_{n}-T_{n}$, which represents the delay difference of the $n$th path seen by the two receivers, is normally much smaller than $1 \mu \mathrm{s}$. Now consider the term $\omega_{1} \tau_{n}$. With the first assumption, and from Fig. 2, we have $c \tau_{n}=d \cos \xi_{n}$, where $c$ is the speed of light and $\xi_{n}$ the angle between the propagation path from the $n$th scatterer and the line $R_{1} R_{2}$. Then, we have $\omega_{1} \tau_{n}=(2 \pi d / \lambda) \cos \xi_{n}$. From Fig. 2, we have $\xi_{n}=\pi-\theta_{n}+\theta_{0}$, where $\theta_{0}$ is the angle between the receivers' direction of motion and $R_{1} R_{2}$. Thus, $\omega_{1} \tau_{n}=-(2 \pi d / \lambda) \cos \left(\theta_{n}-\theta_{0}\right)$. Using (9a), we obtain

$$
\left(\omega_{d_{n}}+\omega_{1}\right) \tau_{n} \simeq-\frac{2 \pi d}{\lambda} \cos \left(\theta_{n}-\theta_{0}\right)
$$

From (6h) and (9b), also with the assumption that $\alpha_{n}=\beta_{n}$, we find that $\mu_{5}$ is given by

$$
\mu_{5}=\frac{A^{2} \omega_{d}^{2}}{2} \times E\left[\sum_{n=1}^{N} \alpha_{n}^{2} \cos \left(\frac{2 \pi d}{\lambda} \cos \left(\theta_{n}-\theta_{0}\right)\right) \cos ^{2} \theta_{n}\right]
$$

Note that we may express $\alpha_{n}^{2}$ as $\alpha_{n}^{2}=p\left(\theta_{n}\right) \mathrm{d} \theta$, the term $p\left(\theta_{n}\right) \mathrm{d} \theta$ representing the fraction of the normalised incoming power within $\mathrm{d} \theta$ of the angle $\theta_{n}$ in the case of a large number $N$ of scatterers, with $p(\theta)$ being the normalised concentration of scatterer power in angle. In the limit $N \rightarrow \infty$, (10a) takes the integral form

$$
\mu_{5}=\frac{A^{2} \omega_{d}^{2}}{2} \int_{0}^{2 \pi} \cos \left(\frac{2 \pi d}{\lambda} \cos \left(\theta-\theta_{0}\right)\right) \cos ^{2} \theta p(\theta) \mathrm{d} \theta
$$

where $p(\theta)$ is the normalised incident power density function at the receivers as function of the angle $\theta$. If the incident power from the scatterers is uniformly distributed, that is, $p(\theta)=1 / 2 \pi$, then after a change of variables in the righthand side of (10b) and a series of simplifications, we arrive at the final result

$$
\begin{aligned}
\mu_{5} & =\frac{\omega_{d}^{2} A^{2}}{4} \times\left\{\left[J_{0}\left(\frac{2 \pi}{\lambda} d\right)-J_{2}\left(\frac{2 \pi}{\lambda} d\right)\right] \cos ^{2} \theta_{0}\right. \\
& \left.+\left[J_{0}\left(\frac{2 \pi}{\lambda} d\right)+J_{2}\left(\frac{2 \pi}{\lambda} d\right)\right] \sin ^{2} \theta_{0}\right\} \\
& =\frac{\omega_{d}^{2} A^{2}}{4} \times\left[J_{0}\left(\frac{2 \pi}{\lambda} d\right)-\cos 2 \theta_{0} J_{2}\left(\frac{2 \pi}{\lambda} d\right)\right]
\end{aligned}
$$

Here, $J_{n}(\cdot)$ is the Bessel function of the first kind of order $n$. Similarly, we can evaluate all the other covariance elements (6a)-(6i) to obtain the following results

$$
\begin{aligned}
& \sigma^{2}=\frac{A^{2}}{2} \\
& \dot{\sigma}_{1}^{2}=\dot{\sigma}_{2}^{2}=\left(2 \pi f_{\mathrm{m}}\right)^{2} \times \frac{\sigma^{2}}{2} \triangleq \dot{\sigma}^{2}
\end{aligned}
$$




$$
\begin{gathered}
\mu_{1}=\sigma^{2} \times J_{0}\left(\frac{2 \pi}{\lambda} d\right) \\
\mu_{2}=\mu_{4}=\mu_{6}=0 \\
\mu_{3}=2 \pi f_{\mathrm{m}} \sigma^{2} J_{1}\left(\frac{2 \pi}{\lambda} d\right) \cos \theta_{0} \\
\mu_{5}=\frac{\left(2 \pi f_{\mathrm{m}}\right)^{2} \sigma^{2}}{2}\left[J_{0}\left(\frac{2 \pi}{\lambda} d\right)-J_{2}\left(\frac{2 \pi}{\lambda} d\right) \cos 2 \theta_{0}\right]
\end{gathered}
$$

From (11a)-(11f), the covariance values between components of the two Rayleigh fading envelopes in our model depend on (i) the maximum Doppler frequency shift $f_{\mathrm{m}}$, (ii) the separation $d$ of two receivers and (iii) the angle $\theta_{0}$ between their direction of motion and the line connecting the two receivers. From the properties of the Bessel functions of the first kind of order $n=0,1,2$, we observe that for a spatial diversity model, as the separation $d$ becomes larger, the covariance matrix $\boldsymbol{\Lambda}$ approaches a diagonal matrix as we expect.

2.3.1 Justification for ignoring $\mu_{3}:$ We can, in principle, derive the pdf $p\left(r_{1}, r_{2}, \dot{r}_{1}, \dot{r}_{2}\right)$ using the covariance matrix $\Lambda$. For this, we need the inverse of the $8 \times 8$ covariance matrix of (8) in closed form. For the spatial diversity model, $\mu_{4}$ is zero by (11d), but $\mu_{3}$ is not always zero. If $\mu_{3}$ is small enough and can be set equal to 0 , we are able to find the inverse of covariance matrix in closed form. The assumption needed is that the absolute value of element $\mu_{3}$ in the covariance matrix $\boldsymbol{\Lambda}$ is very small compared to the diagonal elements in the matrix.

The ratio of $\mu_{3}$ and the diagonal elements $\left(\sigma^{2}, \dot{\sigma}_{1}^{2}, \dot{\sigma}_{2}^{2}\right)$ in the covariance matrix depends on $f_{\mathrm{m}}$ and on $J_{1}(2 \pi d / \lambda) \cos \theta_{0}$, from (11a), (11b) and (11e). One case of interest for which $\mu_{3}=0$ is when $\theta_{0}=\pi / 2$. This means that two receivers are moving in a direction perpendicular to the line connecting them. This is the case for a vehicle with an antenna mounted on each side. In general, if the angle $\theta_{0}$ is close to $\pi / 2$ and the speed is not very high, then $\mu_{3}$ can be assumed to be negligible. In addition, the function $J_{1}(2 \pi d / \lambda)$ has multiple zeros, that is, $\mu_{3}=0$. For any wavelength $\lambda$, there is a sequence of increasing separations $d$ for which $J_{1}(2 \pi d / \lambda)=0$, and therefore $\mu_{3}=0$. For example, at a centre frequency of $1.9 \mathrm{GHz}, \mu_{3}=0$ for a minimum non-zero value of $d=9.6 \mathrm{~cm}$ and for other values of $d$ spaced by $\sim 8 \mathrm{~cm}$ after this.

It is possible to extend the result for the covariance matrix inverse and obtain an approximation that holds when $\mu_{3}$ is not negligible. However, this gives a more complex result, which does not allow for easy further analysis. Note that the condition $\mu_{3}=0$ simplifies the analysis, but is not a design preference.

It is interesting to note that in [9] we also considered the frequency diversity case of a single receiver, and obtained for this case very similar results for the covariance matrix elements. In particular, for frequency diversity the value of $\mu_{3}$ is always 0 , so no further assumptions are needed to the covariance matrix inverse to be established.

\section{Derivation of joint pdf $p\left(r_{1}, r_{2}, \dot{r}_{1}, \dot{r}_{2}\right)$}

In this section, we derive the joint pdf $p\left(r_{1}, r_{2}, \dot{r}_{1}, \dot{r}_{2}\right)$ of the two fading envelopes and their derivatives. We proceed as follows. First, based on the covariance matrix $\boldsymbol{\Lambda}$ for Gaussian variables $\left(X_{1}, Y_{1}, \dot{X}_{1}, \dot{Y}_{1}, X_{2}, Y_{2}, \dot{X}_{2}, \dot{Y}_{2}\right)$, we find the eight-dimensional Gaussian pdf for variables $\left(X_{1}, Y_{1}, \dot{X}_{1}, \dot{Y}_{1}, X_{2}, Y_{2}, \dot{X}_{2}, \dot{Y}_{2}\right)$. By change of variables from Gaussian components of the two Rayleigh fading signals to envelopes, phases and their corresponding derivatives $\left(R_{1}, R_{2}, \dot{R}_{1}, \dot{R}_{2}, \Phi_{1}, \Phi_{2}, \dot{\Phi}_{1}, \dot{\Phi}_{2}\right)$, we obtain the pdf $p\left(r_{1}, r_{2}, \dot{r}_{1}, \dot{r}_{2}, \phi_{1}, \phi_{2}, \dot{\phi}_{1}, \dot{\phi}_{2}\right)$. Finally, by integrating $p\left(r_{1}, r_{2}, \dot{r}_{1}, \dot{r}_{2}, \phi_{1}, \phi_{2}, \phi_{1}, \phi_{2}\right)$ over the phase and phase derivative terms, we obtain the pdf $p\left(r_{1}, r_{2}, \dot{r}_{1}, \dot{r}_{2}\right)$. Note that we use the notation $p\left(r_{1}, r_{2}, \dot{r}_{1}, \dot{r}_{2}\right)$ to represent $p_{R_{1}, R_{2}, \dot{R}_{1}, \dot{R}_{2}}\left(r_{1}, r_{2}, \dot{r}_{1}, \dot{r}_{2}\right)$ and so on. Once the covariance matrix is obtained, the pdf $p\left(x_{1}, y_{1}, \dot{x}_{1}, \dot{y}_{1}, x_{2}, y_{2}, \dot{x}_{2}, \dot{y}_{2}\right)$ can be written down in principle. However, it is difficult to find the inverse of the general $8 \times 8$ covariance matrix $(8)$ in closed form, and even if it could be found the inverse matrix would have fewer zero terms and would be much harder to use. On the other hand, if $\mu_{3}$ is zero, we can obtain the covariance matrix as follows, where we also use the fact that $\dot{\sigma}_{1}^{2}=\dot{\sigma}_{2}^{2} \triangleq \dot{\sigma}^{2}$ for two envelopes with equal power

$$
\boldsymbol{\Lambda}=\left[\begin{array}{cccccccc}
\sigma^{2} & 0 & 0 & 0 & \mu_{1} & \mu_{2} & 0 & 0 \\
0 & \sigma^{2} & 0 & 0 & -\mu_{2} & \mu_{1} & 0 & 0 \\
0 & 0 & \dot{\sigma}^{2} & 0 & 0 & 0 & \mu_{5} & \mu_{6} \\
0 & 0 & 0 & \dot{\sigma}^{2} & 0 & 0 & -\mu_{6} & \mu_{5} \\
\mu_{1} & -\mu_{2} & 0 & 0 & \sigma^{2} & 0 & 0 & 0 \\
\mu_{2} & \mu_{1} & 0 & 0 & 0 & \sigma^{2} & 0 & 0 \\
0 & 0 & \mu_{5} & -\mu_{6} & 0 & 0 & \dot{\sigma}^{2} & 0 \\
0 & 0 & \mu_{6} & \mu_{5} & 0 & 0 & 0 & \dot{\sigma}^{2}
\end{array}\right]
$$

\subsection{Inverse of covariance matrix, $\Lambda^{-1}$}

For the $8 \times 8$ covariance matrix in (12), the inverse is

$$
\boldsymbol{\Lambda}^{-1}=\left[\begin{array}{cccc}
\frac{1}{\sigma^{2}\left(1-\rho^{2}\right)} & 0 & 0 & 0 \\
0 & \frac{1}{\sigma^{2}\left(1-\rho^{2}\right)} & 0 & 0 \\
0 & 0 & \frac{1}{\dot{\sigma}^{2}\left(1-\dot{\rho}^{2}\right)} & 0 \\
0 & 0 & 0 & \frac{1}{\dot{\sigma}^{2}\left(1-\dot{\rho}^{2}\right)} \\
\frac{-\mu_{1}}{\sigma^{4}\left(1-\rho^{2}\right)} & \frac{\mu_{2}}{\sigma^{4}\left(1-\rho^{2}\right)} & 0 & 0 \\
\frac{-\mu_{2}}{\sigma^{4}\left(1-\rho^{2}\right)} & \frac{-\mu_{1}}{\sigma^{4}\left(1-\rho^{2}\right)} & 0 & 0 \\
0 & 0 & \frac{-\mu_{5}}{\dot{\sigma}^{4}\left(1-\dot{\rho}^{2}\right)} & \frac{\mu_{6}}{\dot{\sigma}^{4}\left(1-\dot{\rho}^{2}\right)} \\
0 & 0 & \frac{-\mu_{6}}{\dot{\sigma}^{4}\left(1-\dot{\rho}^{2}\right)} & \frac{-\mu_{5}}{\dot{\sigma}^{4}\left(1-\dot{\rho}^{2}\right)}
\end{array}\right.
$$

$$
\begin{array}{cccc}
\frac{-\mu_{1}}{\sigma^{4}\left(1-\rho^{2}\right)} & \frac{-\mu_{2}}{\sigma^{4}\left(1-\rho^{2}\right)} & 0 & 0 \\
\frac{\mu_{2}}{\sigma^{4}\left(1-\rho^{2}\right)} & \frac{-\mu_{1}}{\sigma^{4}\left(1-\rho^{2}\right)} & 0 & 0 \\
0 & 0 & \frac{-\mu_{5}}{\dot{\sigma}^{4}\left(1-\dot{\rho}^{2}\right)} & \frac{-\mu_{6}}{\dot{\sigma}^{4}\left(1-\dot{\rho}^{2}\right)} \\
0 & 0 & \frac{\mu_{6}}{\dot{\sigma}^{4}\left(1-\dot{\rho}^{2}\right)} & \frac{-\mu_{5}}{\dot{\sigma}^{4}\left(1-\dot{\rho}^{2}\right)} \\
\frac{1}{\sigma^{2}\left(1-\rho^{2}\right)} & 0 & 0 & 0 \\
0 & \frac{1}{\sigma^{2}\left(1-\rho^{2}\right)} & 0 & 0 \\
0 & 0 & \frac{1}{\dot{\sigma}^{2}\left(1-\dot{\rho}^{2}\right)} & 0 \\
0 & 0 & 0 & \frac{1}{\dot{\sigma}^{2}\left(1-\dot{\rho}^{2}\right)}
\end{array}
$$


where

$$
\rho^{2} \triangleq \frac{\mu_{1}^{2}+\mu_{2}^{2}}{\sigma^{4}}, \quad \dot{\rho}^{2} \triangleq \frac{\mu_{5}^{2}+\mu_{6}^{2}}{\dot{\sigma}^{4}}
$$

Note that $\dot{\rho}$ is a notation, which is defined in (14), and not the derivative of $\rho$. From $(11 \mathrm{~b})-(11 \mathrm{~d}),(11 \mathrm{f})$ and (14), we have

$$
\frac{\dot{\rho}^{2}}{\rho^{2}}=\frac{\left[J_{0}((2 \pi / \lambda) d)-J_{2}((2 \pi / \lambda) d) \cos 2 \theta_{0}\right]^{2}}{\left[J_{0}((2 \pi / \lambda) d)\right]^{2}}
$$

We find also that the determinant of the covariance matrix (12) is given by

$$
|\boldsymbol{\Lambda}|=\left[\sigma^{4}\left(1-\rho^{2}\right)\right]^{2}\left[\dot{\sigma}^{4}\left(1-\dot{\rho}^{2}\right)\right]^{2}
$$

\subsection{Pdf $p\left(r_{1}, r_{2}, \dot{r}_{1}, \dot{r}_{2}\right)$}

Once the inverse (13) and the determinant (16) of the covariance matrix are known, the joint pdf $p\left(x_{1}, y_{1}, \dot{x}_{1}, \dot{y}_{1}, x_{2}, y_{2}, \dot{x}_{2}, \dot{y}_{2}\right)$ for the eight Gaussian random variables can be written down. With the transformation of variables from Gaussian components of the two fading channels to the two Rayleigh envelopes $\left(r_{1}, r_{2}\right)$ and phases $\left(\phi_{1}, \phi_{2}\right)$ and their derivatives, we can obtain the joint pdf $p\left(r_{1}, r_{2}, \dot{r}_{1}, \dot{r}_{2}, \phi_{1}, \phi_{2}, \phi_{1}, \phi_{2}\right)$. Finally, we are able to obtain the joint probability density function $p\left(r_{1}, r_{2}, \dot{r}_{1}, \dot{r}_{2}\right)$ by integrating out the $\phi_{1}, \phi_{2}, \dot{\phi}_{1}$ and $\dot{\phi}_{1}$ terms. We have the following main result

$$
\begin{aligned}
p\left(r_{1}, r_{2}, \dot{r}_{1}, \dot{r}_{2}\right)= & \frac{r_{1} r_{2}}{(2 \pi)^{2} \sigma^{4} \dot{\sigma}^{2}\left(1-\rho^{2}\right)} \\
& \times \exp \left\{-\frac{1}{2}\left[\frac{r_{1}^{2}+r_{2}^{2}}{\sigma^{2}\left(1-\rho^{2}\right)}\right]\right\} \\
& \times \int_{0}^{2 \pi} \frac{1}{\sqrt{1-\dot{\rho}^{2} \sin ^{2} \alpha}} \\
& \times \exp \left\{-\frac{1}{2}\left[\frac{-2 r_{1} r_{2}|\rho| \sin \left(\alpha+\phi_{0}-\dot{\phi}_{0}\right)}{\sigma^{2}\left(1-\rho^{2}\right)}\right.\right. \\
& \left.\left.+\frac{\dot{r}_{1}^{2}+\dot{r}_{2}^{2}-2 \dot{r}_{1} \dot{r}_{2}|\dot{\rho}| \sin \alpha}{\dot{\sigma}^{2}\left(1-\dot{\rho}^{2} \sin ^{2} \alpha\right)}\right]\right\} \mathrm{d} \alpha
\end{aligned}
$$

where $r_{1}, r_{2} \in(0,+\infty), \dot{r}_{1}, \dot{r}_{2} \in(-\infty,+\infty)$ and $\phi_{0}, \dot{\phi}_{0}$ are defined as follows

$$
\sin \phi_{0} \triangleq \frac{\mu_{1}}{\sqrt{\mu_{1}^{2}+\mu_{2}^{2}}}, \quad \sin \dot{\phi}_{0} \triangleq \frac{\mu_{5}}{\sqrt{\mu_{5}^{2}+\mu_{6}^{2}}}
$$

From (18), (11c), (11d), and (11f), we have

$$
\begin{aligned}
\phi_{0}-\dot{\phi}_{0}=k \pi, \quad k=0,1 \\
\quad\left(k=0 \text { when } \mu_{1}, \mu_{5} \text { have the same signs }\right)
\end{aligned}
$$

\subsubsection{Envelope cross-correlation coefficient} $\rho_{\text {envelope: }}$ The envelope cross-correlation coefficient between the two Rayleigh fading envelopes $r_{1}$ and $r_{2}$, denoted by $\rho_{\text {envelope }}$, is defined as

$$
\rho_{\text {envelope }}=\frac{\operatorname{Cov}\left(r_{1}, r_{2}\right)}{\sqrt{\operatorname{Var}\left(r_{1}\right)} \sqrt{\operatorname{Var}\left(r_{2}\right)}}
$$

Using the joint pdf $p\left(r_{1}, r_{2}\right)$ (which can be obtained from (17) but is also available in [1]), and tables of integrals [10], we find that this envelope cross-correlation coefficient is (see also [11])

$$
\begin{aligned}
\rho_{\text {envelope }} & =\frac{(\pi / 2) \sigma^{2} F\left(-1 / 2,-1 / 2 ; 1 ; \rho^{2}\right)-(\pi / 2) \sigma^{2}}{(2-(\pi / 2)) \sigma^{2}} \\
& =\frac{\pi}{2} \times \frac{(1 / 2)^{2} \rho^{2}+(1 / 2)^{6} \rho^{4}+(1 / 2)^{9} \rho^{6}+\cdots}{2-(\pi / 2)}
\end{aligned}
$$

where $F\left(-1 / 2,-1 / 2 ; 1 ; \rho^{2}\right)$ is the hypergeometric function. Note that another equivalent expression for the envelope cross-correlation coefficient is given in [1], where the complete elliptic integral is used instead of the hypergeometric function. Note that in (20), the coefficients in front of powers of $\rho$ are decreasing exponentially, and neglecting powers of $\rho$ beyond $\rho^{2}$, we find that $\rho_{\text {envelope }} \simeq \rho^{2}$.

\section{Envelope statistics after diversity combining}

A receiver with MRC will coherently combine the diversity branches by weighting them using the complex conjugate of their respective fading gains and adding them. Thus, the instantaneous signal-to-noise-ratio (SNR) at the output of the combiner is the sum of the SNRs from diversity branches. It is also known [12] that for diversity systems using post-detection product detector combiner, the instantaneous SNR at the output of the combiner equals the sum of SNRs from diversity branches. Therefore the fading channels' effect on performance of these diversity systems can be characterised by the total power. Another diversity combining scheme, called selection combining, is commonly used to avoid the high implementation complexity of the MRC. The diversity branch with maximum received power is selected as the output of this diversity combiner. Therefore the fading channels' effect on performance of such diversity systems can be characterised by the maximum power.

In this section, we study the statistical characteristics of the diversity combined output with MRC (total power) and selection combining (maximum power). In particular, for the case of two correlated equal power fading signals, we derive the distribution and LCR of the envelope arising from each of these two diversity combining schemes. This provides not only the steady-state properties but also transition properties of the equivalent fading channels for the diversity output, and is useful in further work on finite-state Markov models for these cases. On the basis of the results of this section, we can obtain further statistical characteristics such as fading rate and average fade duration, which are related to system characteristics such as handoff and outage probability.

\subsection{Distribution and LCR for total power}

On the basis of the joint pdf $p\left(r_{1}, r_{2}, \dot{r}_{1}, \dot{r}_{2}\right)$ of the two correlated envelopes and their derivatives, the pdf of total power and its derivative can be obtained by a change of variables to square root of total power $r$, angle $\theta$ and their derivatives. Fig. $3 a$ illustrates the transformation. We have

$$
\begin{aligned}
& r_{1}=r \cos \theta \\
& r_{2}=r \sin \theta \\
& r^{2}=r_{1}^{2}+r_{2}^{2}, \quad \text { where } r \geq 0, \quad 0 \leq \theta \leq \pi / 2
\end{aligned}
$$

where $r^{2}$ represents the instantaneous total received signal power from the two Rayleigh fading channels. 

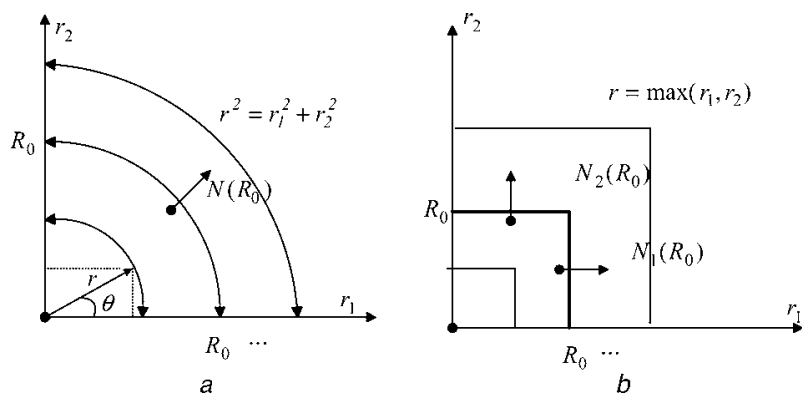

Fig. 3 Illustration of level crossing at level $R_{0}$ for $r$ and variable changes

a $r^{2}=r_{1}^{2}+r_{2}^{2}$ for total power

$b r=\max \left(r_{1}, r_{2}\right)$ for maximum power

From this, we have

$$
\begin{aligned}
& \dot{r}_{1}=\dot{r} \cos \theta-r \sin \theta \dot{\theta} \\
& \dot{r}_{2}=\dot{r} \sin \theta+r \cos \theta \dot{\theta}
\end{aligned}
$$

Using the above transformation, the joint pdf for the new set of variables can be found as

$$
p(r, \dot{r}, \theta, \dot{\theta})=|\boldsymbol{J}| p\left(r_{1}, r_{2}, \dot{r}_{1}, \dot{r}_{2}\right)
$$

where the determinant of the Jacobian $\boldsymbol{J}$ for the transformation can be shown to be $r^{2}$. We can obtain the pdf $p(r, \dot{r})$ from $p(r, \dot{r}, \theta, \dot{\theta})$ by integrating over $\theta$ from 0 to $\pi / 2$ and $\dot{\theta}$ from $-\infty$ to $+\infty$. Using (22) and (17), we obtain the following important result

$$
\begin{aligned}
p(r, \dot{r})= & \frac{\sqrt{2 \pi} r^{3}}{2(2 \pi)^{2} \sigma^{4}\left(1-\rho^{2}\right) \dot{\sigma}} \exp \left\{-\frac{1}{2} \frac{r^{2}}{\sigma^{2}\left(1-\rho^{2}\right)}\right\} \\
& \times \int_{0}^{\pi / 2} \int_{0}^{2 \pi} \frac{\sin 2 \theta}{\sqrt{1+\dot{\rho} \sin 2 \theta \sin \alpha}} \\
& \times \exp \left\{-\frac{1}{2}\left[\frac{-\rho r^{2} \sin 2 \theta \sin \left(\alpha+\phi_{0}-\dot{\phi}_{0}\right)}{\sigma^{2}\left(1-\rho^{2}\right)}\right.\right. \\
& \left.\left.+\frac{\dot{r}^{2}}{\dot{\sigma}^{2}(1+\dot{\rho} \sin 2 \theta \sin \alpha)}\right]\right\} \mathrm{d} \alpha \mathrm{d} \theta
\end{aligned}
$$

where $\rho$ and $\dot{\rho}$ are defined in (14), and $\phi_{0}-\dot{\phi}_{0}$ is given in (19).

From the pdf $p(r, \dot{r})$, we can obtain $p(r)=\int_{-\infty}^{\infty} p(r, \dot{r}) \mathrm{d} \dot{r}$, the steady-state statistics for the total power random process. We use the instantaneous SNR $\gamma=r^{2} / 2 \sigma_{0}^{2}$ from two diversity branches to represent the fading channel condition, where $\sigma_{0}^{2}$ is the additive Gaussian noise variance. From (23), the distribution of $\gamma$ can be obtained as

$$
\begin{aligned}
p(\gamma)= & \frac{8 \gamma}{\gamma_{0}^{2}\left(1-\rho^{2}\right)} \frac{1}{4 \pi} \int_{0}^{\pi / 2} \int_{0}^{2 \pi} \sin \phi \\
& \times \exp \left\{-\frac{1}{2}\left[\frac{4 \gamma(1-\rho \sin \phi \cos \alpha)}{\gamma_{0}\left(1-\rho^{2}\right)}\right]\right\} \mathrm{d} \alpha \mathrm{d} \phi
\end{aligned}
$$

where $\gamma_{0}=4 \sigma^{2} / 2 \sigma_{0}^{2}$ is the average SNR. The pdf $p(\gamma)$ implicitly depends on $\rho_{\text {envelope }}$ through (20) or its approximation $\rho_{\text {envelope }} \simeq \rho^{2}$. We observe the following from (24) (i) when $\rho_{\text {envelope }} \rightarrow 1$, that is the two fading channels are almost identical, the pdf $p(\gamma)$ approaches the pdf of the SNR in a single Rayleigh envelope case, that is the pdf $p\left(\gamma_{1}\right)=1 / \gamma_{10} \exp \left(-\gamma_{1} / \gamma_{10}\right)$ with $\gamma_{1}$ and $\gamma_{10}$ representing the instantaneous and the average SNR for the single Rayleigh envelope (ii) when $\rho_{\text {envelope }} \rightarrow 0$, that is the two fading channels are almost uncorrelated, the pdf $p(\gamma)$ will approach the chi-square pdf with four degrees of freedom: $p(\gamma)=4 \gamma / \gamma_{0}^{2} \exp \left(-2 \gamma / \gamma_{0}\right)$

We now derive the LCR for total power by applying results for level crossings of random processes [13]. The LCR (average number of upcrossings of a level per unit time, or average number of downcrossings of a level per unit time) for the random process $r$ at a given level $r=R_{0}$ is given by

$$
N\left(R_{0}\right)=\left.\int_{0}^{\infty} \dot{r} p(r, \dot{r})\right|_{r=R_{0}} \mathrm{~d} \dot{r}
$$

Using (23), (11b) and carrying out the integral in (25), we obtain

$$
\begin{aligned}
N\left(R_{0}\right)= & \frac{f_{\mathrm{m}}\left(R_{0} / \sigma\right)^{3}}{8 \sqrt{\pi}\left(1-\rho^{2}\right)} \int_{0}^{\pi} \int_{0}^{2 \pi} \sin \phi \sqrt{1+\dot{\rho} \sin \phi \sin \alpha} \\
& \times \exp \left\{-\frac{\left(1-\rho \sin \phi \sin \left(\alpha+\phi_{0}-\dot{\phi}_{0}\right)\right)}{2\left(1-\rho^{2}\right)}\right\} \mathrm{d} \alpha \mathrm{d} \phi
\end{aligned}
$$

For two correlated fading envelopes, the pdf in (24) and the LCR in (26) are expressed in terms of double integrals. However, in the case of two uncorrelated fading envelopes $\left(\rho_{\text {envelope }}=0\right)$, closed form results can be obtained. In particular,

$$
\begin{gathered}
N\left(R_{0}\right)=\frac{\sqrt{\pi}}{2} f_{\mathrm{m}}\left(\frac{R_{0}}{\sigma}\right)^{3} \exp \left[-\frac{1}{2}\left(\frac{R_{0}}{\sigma}\right)^{2}\right] \\
\text { when } \rho_{\text {envelope }}=0
\end{gathered}
$$

From (27), we can find further that the maximum LCR occurs at $R_{0}=3 \sigma$ (or $R_{0} / 2 \sigma=0.866$ ) when two fading channels are uncorrelated. Simulations reported in [7] show very good agreement with the analytical result of (26) for the LCR for total power.

The LCR is in general proportional to the maximum Doppler shift $f_{\mathrm{m}}$ from (26). In Fig. 4, we show the normalised LCR $N\left(R_{0}\right) / 2 \pi f_{\mathrm{m}}$ against the normalised level $R_{0} / 2 \sigma$

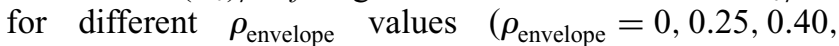
$0.75,0.90)$. Note that the root mean square (rms) value of $r$ is $2 \sigma$. Fig. 4 is an example case where $d / \lambda=1.33$ and

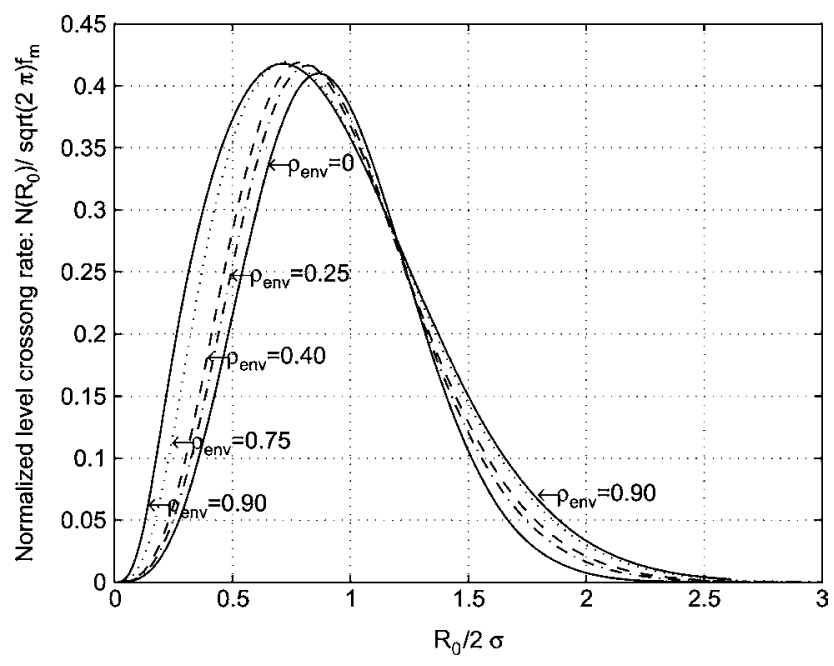

Fig. 4 Normalised $L C R N\left(R_{0}\right) /(2 \pi) f_{m}$ against $R_{0} / 2 \sigma$ for different $\rho_{\text {envelope }}$, total power 
$\theta_{0}=\pi / 2$ in (15), thus $\rho^{2}=0.7908 \dot{\rho}^{2}$. The result is obtained for $k=0$ in (19) (for $\theta_{0}=\pi / 2$, most of time $k=0$, i.e. $\mu_{1}$ and $\mu_{5}$ have the same signs, from numerical calculation). We observe the following: (i) The LCR increases with $\rho_{\text {envelope }}$ in the low level as well as high-level region. This is because as the fading signals become more uncorrelated, their total power is more likely to remain near its mean. (ii) For independent fading envelopes $\left(\rho_{\text {envelope }}=0\right)$, the maximum LCR occurs at $R_{0} / 2 \sigma=0.866$. As an example, suppose $f_{\mathrm{m}}=8.7963 \mathrm{~Hz}$. Then, the LCR $N\left(R_{0}\right)$ is $\sim 8 \mathrm{~s}^{-1}$ at the rms value $2 \sigma$ of the equivalent received envelope $r$. In general, the LCR reaches a maximum near $R_{0} / 2 \sigma \simeq 0.7$. (iii) For $\rho_{\text {envelope }}$ approaching 1 , the LCR for total power approaches the result of the LCR for a single channel [2].

Given a SNR level $\gamma=\Gamma_{0}$, the average duration of fades is the ratio of the total time the SNR is below $\Gamma_{0}$ and the total number of fades, both measured during a certain large time interval $T$. Let $\boldsymbol{\tau}$ be the average duration of fades and $\boldsymbol{\tau}_{i}$, the duration of each fade. Then $\boldsymbol{\tau}=\sum \boldsymbol{\tau}_{i} /\left(N\left(\Gamma_{0}\right) T\right)$. The ratio $\sum \boldsymbol{\tau}_{i} / T$ corresponds to the probability that $\gamma$ is below $\Gamma_{0}$. Hence

$$
\boldsymbol{\tau}=\frac{1}{N\left(\Gamma_{0}\right)} \operatorname{Prob}\left[\gamma \leq \Gamma_{0}\right]=\frac{1}{N\left(\Gamma_{0}\right)} \int_{0}^{\Gamma_{0}} p(\gamma) \mathrm{d} \gamma
$$

where $p(\gamma)$ is given by (24). $N\left(\Gamma_{0}\right)$ can be obtained from (26) and is given by

$$
\begin{aligned}
N\left(\Gamma_{0}\right)= & \frac{f_{\mathrm{m}}\left(\sqrt{4 \Gamma_{0} / \gamma_{0}}\right)^{3}}{8 \sqrt{\pi}\left(1-\rho^{2}\right)} \int_{0}^{\pi} \int_{0}^{2 \pi} \sin \phi \sqrt{1+\dot{\rho} \sin \phi \sin \alpha} \\
& \times \exp \left\{\frac{-\left(1-\rho \sin \phi \sin \left(\alpha+\phi_{0}-\dot{\phi}_{0}\right)\right)}{2\left(1-\rho^{2}\right)} \times \frac{4 \Gamma_{0}}{\gamma_{0}}\right\} \mathrm{d} \alpha \mathrm{d} \phi
\end{aligned}
$$

where $\gamma_{0}=4 \sigma^{2} / 2 \sigma_{0}^{2}$ is the average SNR.

\subsection{Distribution and LCR for maximum power}

In a diversity system with selection combining, the maximum power from different diversity branches determines the diversity system performance [5]. Therefore the fading channels' effect on the performance of selection diversity can be characterised by the maximum power. On the basis of the joint pdf $p\left(r_{1}, r_{2}, \dot{r}_{1}, \dot{r}_{2}\right)$ of two correlated envelopes and their derivatives, we can obtain the distribution of the maximum-power random process and its LCR characteristic. Let $r=\max \left(r_{1}, r_{2}\right)$, where $r_{1}$ and $r_{2}$ are the envelopes of the two received correlated Rayleigh fading signals. From (17) or [1], we can obtain the pdf $p\left(r_{1}, r_{2}\right)$ of the two correlated fading envelopes. Then, we find the distribution function for the maximum to be

$$
P(r)=\int_{0}^{r} \int_{0}^{r} p\left(r_{1}, r_{2}\right) \mathrm{d} r_{1} \mathrm{~d} r_{2}
$$

Let $\gamma=r^{2} / 2 \sigma_{0}^{2}$ be the instantaneous SNR for maximum power diversity combining (where $\sigma_{0}^{2}$ is the variance of additive Gaussian noise). From (30), the pdf $p(\gamma)$ of the
SNR can be obtained as

$$
\begin{aligned}
p(\gamma)= & \frac{1}{\pi \gamma_{1_{0}}} \int_{0}^{\sqrt{\left(2 \gamma / \gamma_{1_{0}}\left(1-\rho^{2}\right)\right.}} \int_{0}^{2 \pi} y \exp \left\{-\frac{1}{2}\left[y^{2}-2 y \rho\right.\right. \\
& \left.\left.\times \sin \theta \sqrt{\frac{2 \gamma}{\gamma_{1_{0}}\left(1-\rho^{2}\right)}}+\frac{2 \gamma}{\gamma_{1_{0}}\left(1-\rho^{2}\right)}\right]\right\} \mathrm{d} \theta \mathrm{d} y
\end{aligned}
$$

where $\gamma_{10}$ is the average SNR for the first channel, that is $\gamma_{1_{0}}=2 \sigma^{2} / 2 \sigma_{0}^{2}$. When $\rho_{\text {envelope }}$ approaches 1 , which means $\rho \rightarrow 1$ from (20), the pdf $p(\gamma)$ becomes the pdf of the SNR for a single Rayleigh envelope.

Note that in [5], the pdf of maximum power was obtained by a different approach from ours, and we can show that the two results are equivalent. We can also find the average maximum power to be $2 \sigma^{2}\left(1+\left(1-\rho^{2}\right) / 2\right)$, where $2 \sigma^{2}$ is the average power for a single channel. As noted earlier, the average error probability performance with selection combining has been investigated [14]. However, [14] does not consider a dynamic channel model and therefore does not characterise functions such as the LCR.

We now derive the LCR for maximum power based on the pdf $p\left(r_{1}, r_{2}, \dot{r}_{1}, \dot{r}_{2}\right)$ of two correlated envelopes and their derivatives. Fig. $3 b$ illustrates the (positive direction only) level crossings of the maximum envelope $r$ at level $r=R_{0}$. The LCR $N\left(R_{0}\right)$ consists of two components $N_{1}\left(R_{0}\right)$ and $N_{2}\left(R_{0}\right)$ as shown in the figure

$$
N\left(R_{0}\right)=N_{1}\left(R_{0}\right)+N_{2}\left(R_{0}\right)
$$

$N_{1}\left(R_{0}\right)$ represents the crossing rate due to the event $\left\{r_{1}\right.$ crosses the boundary $r_{1}=R_{0}$ conditioned on $\left.r_{2}<R_{0}\right\}$. $N_{2}\left(R_{0}\right)$ represents the crossing rate due to the event $\left\{r_{2}\right.$ crosses the boundary $r_{2}=R_{0}$ conditioned on $\left.r_{1}<R_{0}\right\}$. On the basis of standard results for level crossings of a random process [13], we have

$$
N_{1}\left(R_{0}\right)=\left.\int_{0}^{\infty} \int_{0}^{R_{0}} \dot{r}_{1} p\left(r_{1}, r_{2}, \dot{r}_{1}\right)\right|_{r_{1}=R_{0}} \mathrm{~d} r_{2} \mathrm{~d} \dot{r}_{1}
$$

where the marginal pdf $p\left(r_{1}, r_{2}, \dot{r}_{1}\right)$ can be obtained from $p\left(r_{1}, r_{2}, \dot{r}_{1}, \dot{r}_{2}\right)$ given by (17). Similarly, we can obtain $N_{2}\left(R_{0}\right)$. From (32) and (33), we can obtain the following LCR for the maximum envelope

$$
\begin{aligned}
N\left(R_{0}\right)= & \frac{2 f_{\mathrm{m}} \sqrt{\pi}}{\pi\left(1-\rho^{2}\right)} \frac{R_{0}}{\sigma^{3}} \int_{-\pi / 2}^{\pi / 2} \int_{0}^{R_{0}} r_{2} \\
& \exp \left\{-\frac{1}{2}\left[\frac{r_{2}^{2}-2 \rho r_{2} R_{0} \sin \alpha+R_{0}^{2}}{\sigma^{2}\left(1-\rho^{2}\right)}\right]\right\} \mathrm{d} r_{2} \mathrm{~d} \alpha
\end{aligned}
$$

Further, we obtain for the LCR of the received instantaneous SNR ratio $\gamma=r^{2} / 2 \sigma_{0}^{2}$ at level $\gamma=\Gamma_{0}$

$$
\begin{aligned}
N\left(\Gamma_{0}\right)= & \frac{2 f_{\mathrm{m}} \sqrt{\pi}}{\pi\left(1-\rho^{2}\right)} \sqrt{\frac{2 \Gamma_{0}}{\gamma_{1_{0}}}} \int_{-\pi / 2}^{\pi / 2} \int_{0}^{\left.\sqrt{\left(2 \Gamma_{0} / \gamma_{1_{0}}\right.}\right)} x \\
& \exp \left\{-\frac{1}{2}\left[\begin{array}{c}
x^{2}-2 \rho x \sqrt{\left(2 \Gamma_{0} / \gamma_{1_{0}}\right)} \\
\frac{\sin \alpha+\left(2 \Gamma_{0} / \gamma_{1_{0}}\right)}{1-\rho^{2}}
\end{array}\right]\right\} \mathrm{d} x \mathrm{~d} \alpha
\end{aligned}
$$

where $\gamma_{1_{0}}=2 \sigma^{2} / 2 \sigma_{0}^{2}$ is the average SNR of one channel. In Fig. 5, we show the normalised LCR $N\left(R_{0}\right) /(2 \pi) f_{\mathrm{m}}$ against the normalised level $R_{0} / 2 \sigma$ for different $\rho_{\text {envelope }}$ values. We observe that for maximum power selection combining, an increase in correlation leads to an increase in the LCR at 


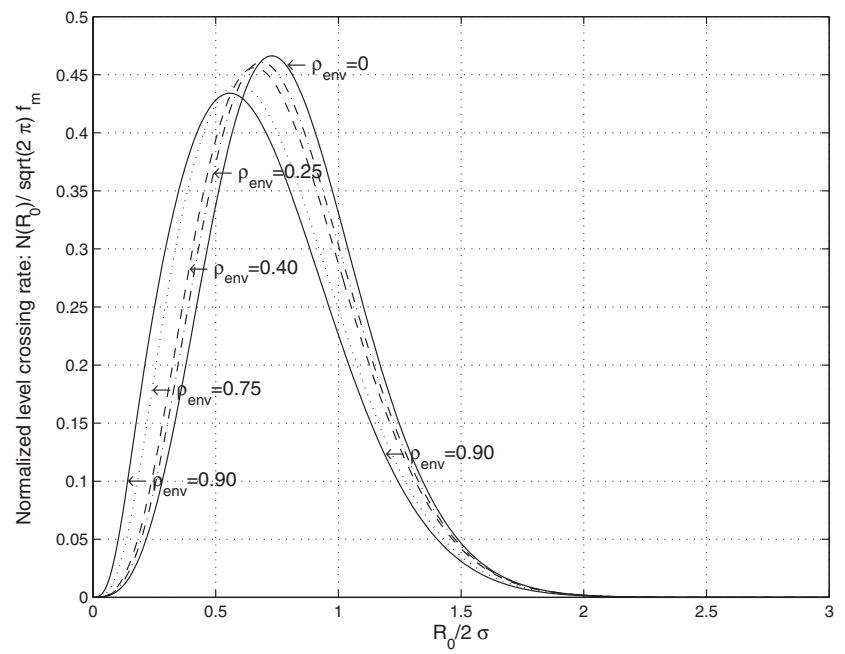

Fig. 5 Normalised LCR $N\left(R_{0}\right) /(2 \pi) f_{m}$ against $R_{0} / 2 \sigma$ for different $\rho_{\text {envelope }}$, maximum power

low levels and to a reduction in the LCR at high levels. Simulations reported in [9] show very good agreement with the analytical result of (35) for the LCR for maximum power. In the case of two uncorrelated fading envelopes $\left(\rho_{\text {envelope }}=0\right)$, the pdf in (31) and LCR in (35) become the following

$$
\begin{aligned}
p(\gamma) & =\frac{2}{\gamma_{1_{0}}} \exp \left[-\frac{\gamma}{\gamma_{1_{0}}}\right]\left(1-\exp \left[-\frac{\gamma}{\gamma_{1_{0}}}\right]\right) \\
N\left(\Gamma_{0}\right) & =2 \sqrt{\pi} f_{\mathrm{m}} \sqrt{\frac{2 \Gamma_{0}}{\gamma_{1_{0}}}} \exp \left[-\frac{\Gamma_{0}}{\gamma_{1_{0}}}\right]\left(1-\exp \left[-\frac{\Gamma_{0}}{\gamma_{1_{0}}}\right]\right)
\end{aligned}
$$

As in the total power case, the average duration $\tau$ of fades for maximum power can be obtained using (28), with $p(\gamma)$ given by (31) and $N\left(\Gamma_{0}\right)$ given by (35).

\section{Conclusion}

We have built a physical multipath model to represent two correlated Rayleigh fading channels in spatial diversity reception. We have explored and evaluated the covariance matrix elements for the Gaussian components and their time derivatives in the description of the two Rayleigh fading envelopes. The joint pdf of the two correlated fading envelopes and their derivatives was derived, and from this we obtained other statistical characteristics of interest. For the total power in MRC diversity combining, we obtained the joint pdf $p(r, \dot{r})$ for total power and its derivative, as well as the LCR. This allows both static and dynamic characterisations for a wireless link. Corresponding results on the distribution and LCR for maximum power (selection combining) were also given. It is possible to obtain useful characteristics such as average duration of fades, fading rate, outage probability and so on from these results. In addition to the results we have given in this paper, we have applied our considerations for the more general case of two correlated envelopes with unequal powers, as well as the case of multiple uncorrelated envelopes. These results have been presented in [9].

The statistical characteristics we have given for total power and maximum power have been validated through simulations of fading channels as reported our previous works [7, 9]. An important application of our results is in the design of finite-state Markov models representing correlated Rayleigh fading channels in diversity systems; we have used such models quite successfully to analyse the performance of ARQ schemes [7,9]. The results of this paper are also broadly useful in understanding the origin and strength of correlation between diversity channels and its specific effects on performance. In general, these results provide means for performance analysis of diversity schemes in correlated fading channels and reduce dependence on bit-level simulations.

\section{References}

1 Jakes, W.C.: 'Microwave mobile communications' (IEEE Press, Piscataway, NJ, 1993)

2 Yacoub, M.D.: 'Foundations of mobile radio engineering' (CRC, Boca Raton, FL, 1993)

3 Zhang, Q., and Kassam, S.A.: 'Final-state Markov model for Rayleigh fading channels', IEEE Trans. Commun., 1999, 47, (11), pp. 1688-1692

4 Zhang, Q., and Kassam, S.A.: 'Hybrid ARQ with selective combining for fading channels', IEEE J. Sel. Areas Commun., 1999, 17, pp. $867-880$

5 Ko, Y., Alouini, M., and Simon, M.K.: 'Average SNR of dual selection combining over correlated Nakagami-m fading channels', IEEE Commun. Lett., 2000, 4, (1), pp. 12-14

6 Ertel, R.B., and Reed, J.H.: 'Generation of two equal power correlated Rayleigh fading envelopes', IEEE Commun. Lett., 1998, 2, (10), pp. $276-278$

7 Tang, W., and Kassam, S.A.: 'Finite-state Markov models for total power of two correlated Rayleigh fading channels'. 2002 Conf. on Information Sciences and Systems, Princeton University, March 20-22, 2002, pp. Wp7-1-Wp7-6

8 Dong, X., and Beaulieu, N.C.: 'Average level crossing rate and fade duration of maximal ratio diversity in unbalanced and correlated channels'. Proc. Wireless Communications and Networking Conf., WCNC 2002, March 2002, vol. 2, pp. $762-767$

9 Tang, W.: 'Finite-state Markov models for correlated Rayleigh fading channels in wireless communications', $\mathrm{PhD}$, Dissertation, Electrical Engineering Department, University of Pennsylvania, 2001

10 Gradshteyn, I.S., and Ryzhik, I.W.: 'Table of integrals, series and products' (Academic, New York, 1965)

11 Stuber, G.L.: 'Principles of mobile communication' (Kluwer Academic Publishers, Norwell, MA, 2001, 2nd edn.)

12 Proakis, J.G.: 'Digital communications' (McGraw-Hill, Inc., New York, 1995, 3rd edn.)

13 Cramèr, H., and Leadbetter, M.R.: 'Stationary and related stochastic processes' (John Wiley \& Sons, Inc., New York, 1967)

14 Simon, M.K., and Alouini, M.: 'A unified performance analysis of digital communication with dual selective combining diversity over correlated Rayleigh and Nakagami-m fading channel', IEEE Trans. Commun., 1999, 47, (1), pp. 33-43 